\title{
Context-Aware Emergency Messaging System Framework Utilizing Social Relations as Services
}

\author{
Regin Cabacas ${ }^{1}$, Ravi Sankar ${ }^{2}$ and In-Ho $\mathrm{Ra}^{1 *}$ \\ ${ }^{1}$ Kunsan National University, Kunsan, South Korea \\ ${ }^{2}$ University of South Florida, Tampa, Florida, USA \\ rcabacas@kunsan.ac.kr,sankar@usf.edu,ihra@kunsan.ac.kr

\section{Abstract}

Emergency alert and messaging systems are commonly enployed to slerve users when emergency situation occurs. Emergency situations requne utmost importance in terms of nearness, speed and accuracy of response. However, most of the researches in literature are incapable of deriving accurate and efficient response to a user query leaving most of solutions to be location based. We see that utilization of user's context information and social network relations can further improve emergency alert systems. With this opportunity, we proposed an approach that utilizes important context information to provide accurate and fast response to an occurring emergenct. This proposed framework is applicable for both low (e.g. flat tire and vehicle repair) and high domain danger types (e.g. fire and natural calamities) emergency situation Within its architecture, we identify several key factors such as participatory scheme, context management, and service management with decision support rules that enable the development of an efficient and practical framework for emergency situations.

Keywords: contert-aware, emergengy systems, context-aware framework, social relations

\section{Introduction}

According to Amerigan Red Cross, "Social Media in Disasters and Emergencies", 18 percent out of 1,058 adults surveyed turn to digital social media if calls to 911 were unsuccessful. Sixty nune percent said emergency response agencies should regularly monitor their Web sttes and social media networks so they can respond promptly to requests; 74 percent said they expect help to arrive within an hour [1].

More and more people are turning to social networks not only in getting up to date infermation but also in asking for help when traditional emergency response systems are unavailable [2]. However, the role of social network in emergency situations has been limitedly used as most services don't make good use of contextual information. The proposed framework aims to exploit social network relations (family, friends, neighbors, etc.) as possible services when emergency occur.

In most context-aware applications, processing of service queries isolates various forms of user "preference" or "context". For example, when a user send queries on "safest" taxi service, existing query processors reduce the meaning of "safest" to "closest" making most of context-aware applications act as location based services.

*Corresponding Author 
However, aside from location, there is a need to utilize other context-aware concepts such as time, preferences, activity, behavior and other sensed data. For example, when a user send queries on "safest" taxi service, existing query processors reduce the meaning of "safest" to "closest" making most of context-aware applications act as location based services. However, aside from location, there is a need to utilize other context-aware concepts such as time, preferences, activity, behavior and other sensed data.

This paper aims to provide a framework for emergency messaging systems using context information and social relations as services. It also aims to improve the state of help queries for emergency transport services, troubleshooting, repair and other maintenance jobs, medical and other professional help by giving users seyeral alternatives based on the query and user's context. In Section 2, we present several existing context-aware frameworks. Section 3 discussed the motivating scenario for the proposed framework and the system architecture is shown in Section 4. Section 5 addresses major concepts incorporated in the framework and lastly, Section 6 concludes and presents future works.

\section{Related Work}

Recently, several frameworks have been proposed and implemented for contextaware services. However, only a few existing research works are for emergency domains.

\section{- Mona Emergency Systems (MES),}

MES is an emergency messaging system that has a design enhancing the flow of information that will be transferred to the actors within the system. In MES spatial relations are used to defermine relevance of messages. It works with real-time contextual information that is collected by the system where information goes through a Disaster Management Unit which analyzes the spatial relations between the system elements to provide the right message at the right time [3].

\section{- Hermes}

Hermes is a next-generation toolkit and framework for developing context-aware applications in mobile (environment. Hermes promotes a context oriented programming model that divides applications into components (i.e., widgets), each handles a single type of context [4].

\section{- Context Toolkit}

Context Toolkit is a context-aware framework that uses a simple attribute-value tuple as it context model which describes a widget based toolkit for context-aware application development, originally designed for a static smart home environment. It usually requires a special lab-like environment and specific hardware for implementation [5].

\section{Motivating Scenario}

Existing distress messaging systems do not utilize important contextual information that can be derived from the user's message. For example a flat tire situation for a specific user; existing distress messaging systems only take into account the distance of available services in response to the user's query. In this example of flat tire scenario, response to such query would likely receive a "change of tire" response with closest service available in the user's 
current location. In certain situations, this might not be the best solution. Let's take a look at a particular message, "Flat tire in Rosewood St., Important meeting at Ocean Bay 9pm. Help!" Taking into account the time (given by the message timestamp), location (distance of user, its activity, and services), and a context-aware applications should give the user several options such as taxi service, fetch by a nearby friend or change of tire. In the proposed framework, we provide options that can be ranked according to the relevance of the solution to the problem calculated by the inferred contextual information from a message along with important information such as service (name, contact info.), distance from the location, time to travel and best route or alternative route. Figure 1 represents the possible application domain or service model of the proposed framework.

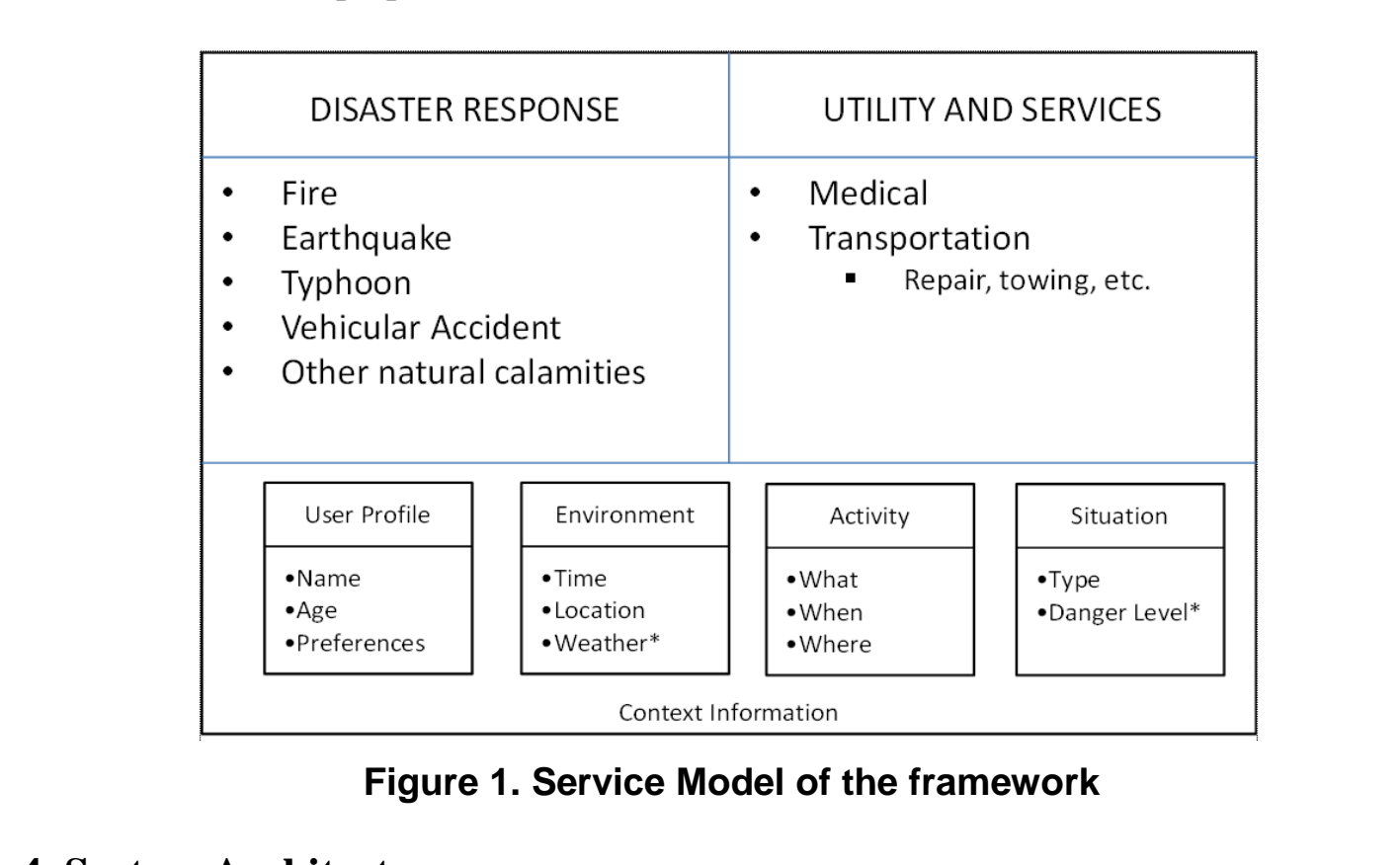

\section{System Architecture}

The implementation of the whole architecture is assumed to be a service subscribed function. A user to aval the service must register to the system and the system stores a profile of each user. In that manner, the system works in a participatory way where services and users are considered as a part of user's social connections which could be respondent to a particular user s query.

The system architecture is divided into two components; context manager and service manager Context information which is collected, processed and inferred by the context manager can be classified into three major contexts such as environment (location, time, weather), activity (where, when and why) and user context which is basically stored in the User Profiles. Once a task is established by the context manager, a trigger will load a task and dispatch it to the service manager. And then service manager identifies and ranked possible services for the receiver based on user's context. 


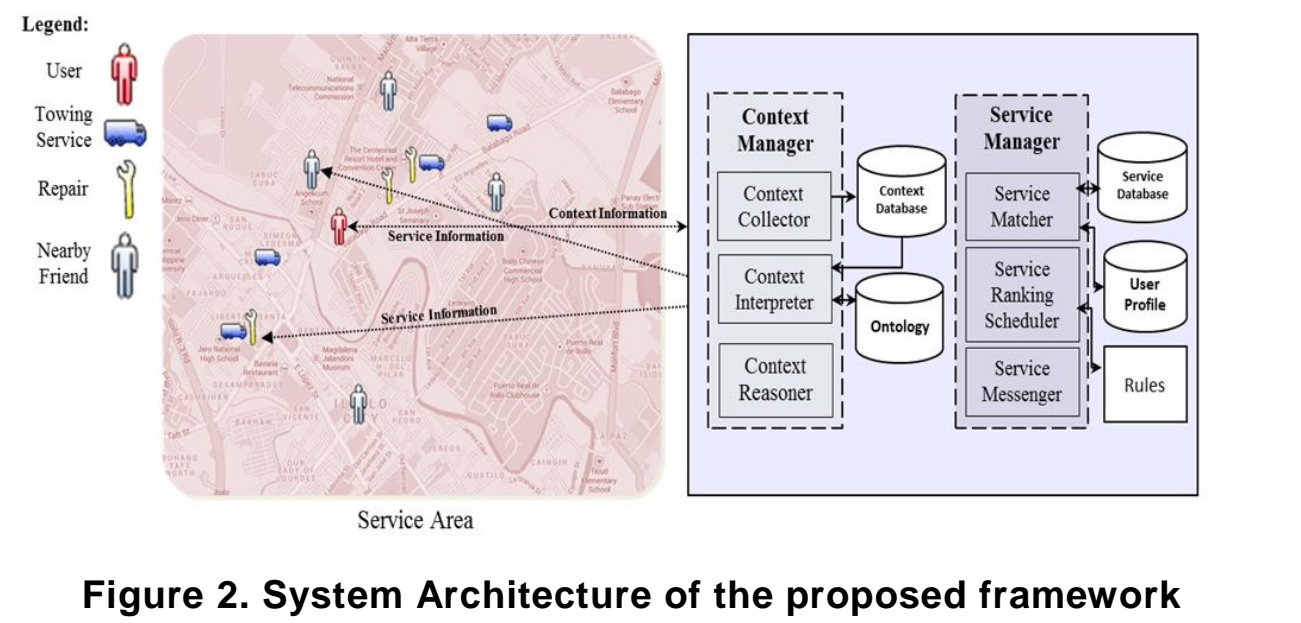

As shown in Figure 2, the system has several features that support context information reflecting content centric and people-centric design concept that utilizes social network relations as plausible query response.

\section{System Concepts}

The following subsections disclosed severat important concepts that is incorporated in the proposed framework.

\subsection{Participatory Scheme}

The proposed system employs participatory approach where subscribers' information (local services, friends and experts such as doctors and mechanic) is stored in the service database and utilized for petforming dedicated services. This can build a basis in determining probable receiyers or responders of the query.

Social networking sites (e.g., Facebook), microblogging services (e.g., Twitter) and content-sharing sites Q8., YouTube and Flickr) have introduced the opportunity for wide-scale, online socyal participation [6]. Participation may be in the form of formal subscription to social network applications where users are required to divulge certain personal infornation or to be voluntary just like surveys.

\subsection{Confext Manager}

Context Manager provides requisite context information extracted from the information stored in the context database. Context Manager includes several components, such as Context Collector, Context Interpreter and Context Reasoner.

Context collector gathers important user's context information. Context collector is responsible for collecting and storing context from user's distress messages and serializing descriptions into a form amenable for direct input into Context Interpreter. Since the distress message has diverse chronological features and data formats, raw data needs to be converted to meaningful data. This can be done by ontology application (such as OWL (Web Ontology Language)) [5] which has capability to specify characteristics and functionalities of all sources of information; this is one of the important functions of the Context interpreter. Figure 3 shows general ontology concept. 


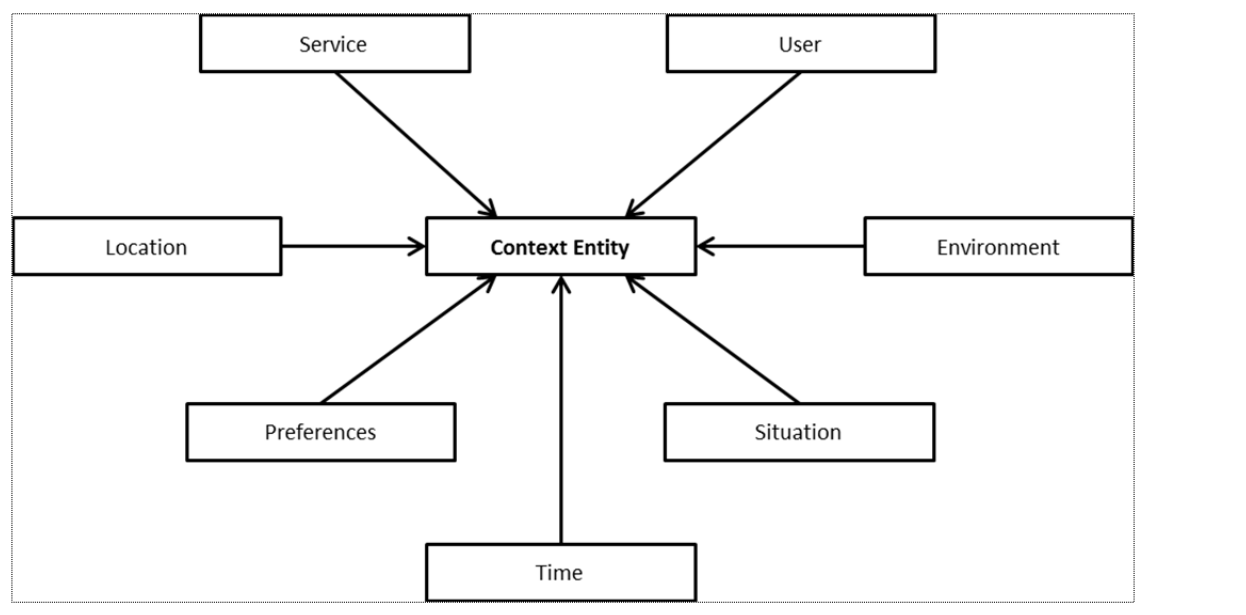

Figure 3. General Context Ontology

Context Reasoner acts as a context provider. It provides high-level contexts by interpreting low-level contexts. In general, dow-level context is simple and can be directly acquired from sensors or other sources (locationdime) while high-level context is abstract and inferred from a piece of low level context (i.e., situation, danger type) [7]. Authors in [7], provide several techniques for inferring high level context out of collected low-level context. Techniques wouldinchude but are not limited to Classifiers, Filters and Context Integration. Figure 4 illustrates the interaction of each context.

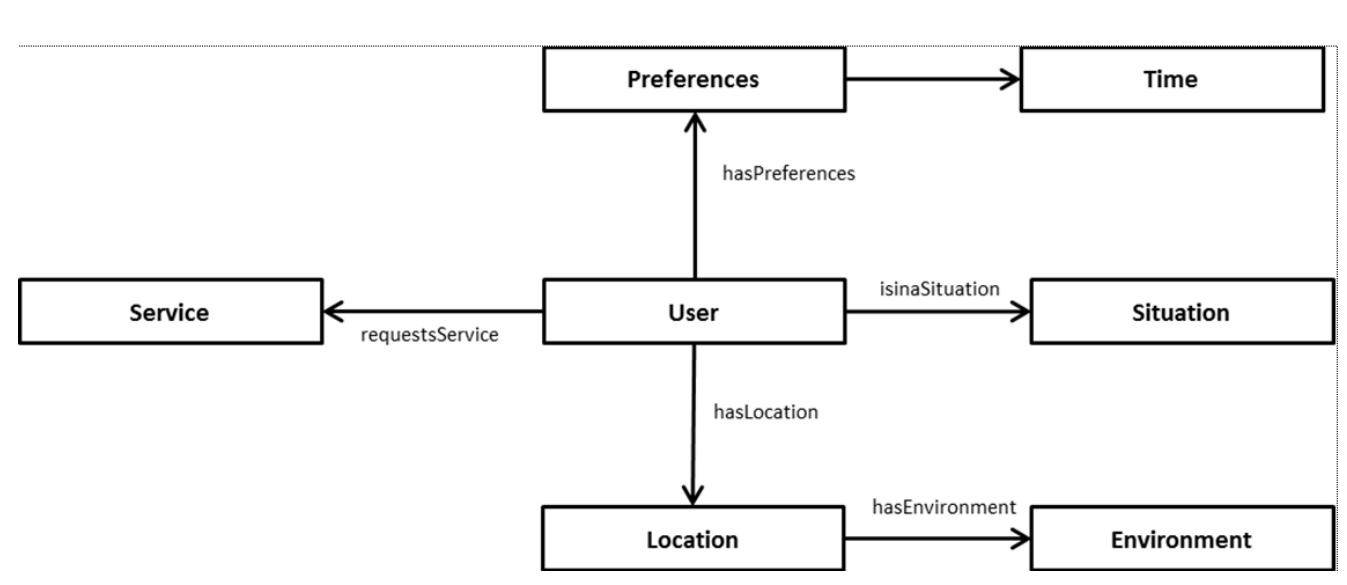

Figure 4. User Context Ontology

\subsection{Service Manager}

Service manager has three modules: service matcher, service ranking scheduler and service messenger.

\section{A. Service Matcher}

Service matcher implements simple straightforward matching algorithm. Since other users (including nearby friends or professional experts) are considered as services in the system, this module matches the available users in the vicinity to the user's location as a service. Algorithm 1 and 2 show that how matching process for a single query is done in this module. 


\section{Algorithm 1: Function Service Lister}

Input: situation (context inferred by the Context Reasoner)

Output: service_list

For all services in the service database do

If the required service type matches to the pre-defined situation then

Add the service to service_list

Else

Increment service index

End if

End for

\section{Algorithm 2: Function UserAsService Lister}

Input: userNeeded (context inferred by the Context Reasoner)

Output: user_as_services_list

For all active_user's indices in the usen database do

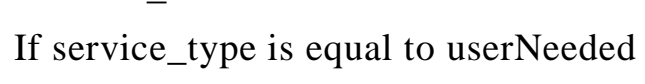

Else Oncrementuset's index

Add userto user_as services_list

End if

End for

\section{B. Service Ranking Scheduler}

This module is responsible for the service relevance ranking of the candidate services provided by the Service Matcher. We take into account the location, time and situation for the ranking function. A ranking function proposed by [8] which utilizes some of our context information in ranking objects is adopted for this module.

\section{- Location Ranker}

The Location Ranker is an evaluation function for a user's location compared to that of the given candidate service. All services exceeding the most appropriate distance (threshold $\theta\left(u_{i}\right)$ ) which is based on the user's preference are discarded from the list. The ranking value of the location ranker is computed by a generic equation as follows: 


$$
\operatorname{loc}\left(S_{i}, \theta\left(u_{i}\right)\right)=\left\{\begin{array}{l}
1 \\
0,
\end{array}-\frac{\| \text { distance }\left(S_{i}, u_{i}\right)-\Theta\left(u_{i}\right) \|}{\Theta\left(u_{i}\right)}, \text { distance }\left(S_{i}, u_{i}\right)<\Theta\left(u_{i}\right)\right.
$$

The location ranker serves as a filter criterion and hence is multiplied with other rankers as all rankers are normalized to a continuous interval between 0 and 1 . The following equation shows the general ranking function for a given service instance si depending on the preferences of a specific user u:

\section{- Time Ranker}

The time ranker [8] works in similar to the location ranker with the difference that a certain service is not discarded because of its temporal properties. This is anevaluation function of the temporal distance between the candidate service and the user. The ranking value of the time ranker is computed as follows:

$$
\operatorname{time}\left(S_{i}, \tau\left(u_{i}\right)\right)=\left\{\begin{array}{l}
1 \\
0,
\end{array}-\frac{\| \operatorname{time}\left(S_{i}, u_{i}\right)-\tau\left(u_{i}\right) \mid}{\tau\left(u_{i}\right)}\right.
$$

The overall ranking is calculated as follows:

\section{Service Messenger}

$$
R_{u}\left(s_{i}\right)=\alpha_{u} \cdot \operatorname{loc}\left(\delta_{i j},\left(u_{i}\right)\right), \beta_{u} \operatorname{time}\left(s_{i}, \tau\left(u_{i}\right)\right)
$$

The Service Messenger works in two-way; broadcast messaging and user-defined messaging according to the situation of a query. When the Service Ranking identifies the most appropriate service, Service Messenger will dispatch each message to the corresponding services about the query if danger level is high (fire, earthquake, etc.) [9]. However, for low dangen lever (such as flat tire, battery out) the services in ranked list with detaled information (distance, ETA) can be utilized as option while as the user's final choice is more suitable for taking better service in the current situation.

\section{Conclusion}

Context-awareness captures and represents the user's physical and social environments Moreover, in the development of richer applications, this information can be used to enhance query-service systems especially during emergency situations. The proposed framework is designed to improve the accuracy and speed of service response to emergency queries by utilizing context information and social relations as services. The framework is designed two have two modules that handles processing of context information and identification and ranking of services with regards to the user query. It also aims to be applicable with other services such as tour management, traffic management and dynamic route planner.

As future work, we seek to implement the proposed framework and utilize Twitter as messaging platform for evaluating user query since there are a good number of distress messages that can be obtained from this microblogging site. Also, we aim to add service area visualization with important details of every ranked service in the implementation. 


\section{Acknowledgements}

This research was supported by Basic Science Research Program through the National Research Foundation of Korea (NRF) funded by the Ministry of Education, Science and Technology (2013054460)

\section{References}

[1] C. Jun, "Key Issues and Research Directions in GIS's Spatial Data Models", Journal of Geographical Sciences, vol. S1, (1995), pp. 24-33.

[2] B. Schilit, N. Adams and R. Want, Editors, "Context-Aware Computing Applications. Proceedings of IEEE Workshop on Mobile Computing Systems and Applications", (1994), pp. 85-90.

[3] A. Almagrabi, S. W. Loke and T. Torabi, "Towards context-aware messaging for emergency situations", Proceedings of 2012 International Symposium on Communications and Information Technologies (ISCIT), (2012), pp. 116-121.

[4] S. Buthpitiya, F. Luqman, M. Griss, Bo Xing and A.K Dey Hermes - A Context-Aware Application Development Framework and Toolkit for the Mobile Etvironment", Proceedings of the 26th International Conference on Advanced Information Networking and Applications Workshops (WAINA), (2012), pp. 663670.

[5] S. De and K. Moessner, "A framework for nobile, context^aware applications", Proceedings of the International Conference on Telecommunications, (2009), pp. 232-237.

[6] D. Burke, M. Estrin, A. Hansen, N. Parker, S. Ramanathan and M. B. Srivastava, "Participatory Sensing", Proceedings of the ACM Sensys World(Sensør Web Workshop, (2006), pp. 1-4.

[7] D. Riboni, L. Pareschi and C. Bettihi, Towards the adaptive integration of multiple context reasoners in pervasive computing environments", Proceedings of the 8th IEEE International Conference on Pervasive Computing and Communications Workshops (PERCOM Workshops), (2010), pp.25-29.

[8] A. Emrich, A. Chapko, D. Werth and P. Loos, "Adaptive, Multi-criteria Recommendations for LocationBased Services", Proceedings of the 46th Hawaii International Conference on System Sciences (HICSS), (2013), pp.1165-1173.

[9] A. Aloudat and Y. Alzoubi, "Rankings of importance of location-based services utilization for emergency management, Prodeedings of the 2010 IEEE International Symposium on Technology and Society (ISTAS), (2010), pp. 406-410.
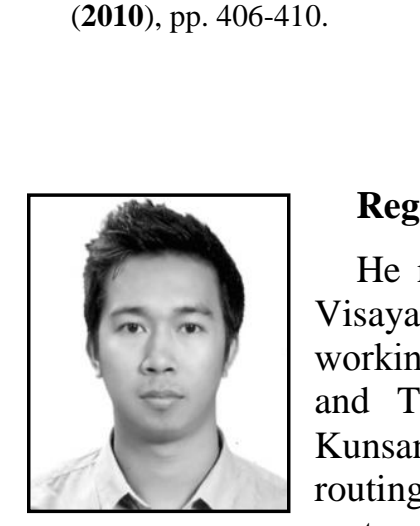

\section{Regin Cabacas}

He received the B.S. degree in Information Technology from West Visayas State University, Iloilo, Philippines, in 2010. He is currently working toward the Master's degree with the Department of Information and Telecommunications Engineering, Kunsan National University, Kunsan, South Korea. His research interests include energy efficient routing in delay tolerant networks, social network analysis, recommender systems, mobile opportunistic sensor networks and integration of sensor networks and social networks. 


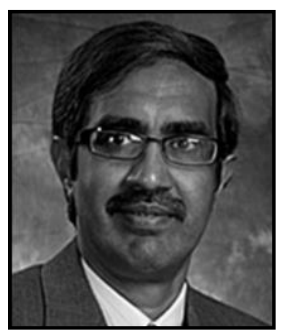

\section{Ravi Sankar}

$\mathrm{He}$ received the B.E. (Honors) degree in electronics and communication engineering from the University of Madras, Chennai, India in 1978, the M. Eng. degree in electrical engineering from Concordia University, Montreal, QC, Canada in 1980, and the Ph.D. degree in electrical engineering from Pennsylvania State University, USA in 1985.

Since then, he has been with the Department of Electrical Engineering, University of South Florida, Tampa, Florida, USA. He is currently a Professor of electrical engineering, Supervisor of the Communications and Signal Processing Graduate Program Track, member of the Biomedical Engineering Program, and Director of the Interdisciplinary Communications, Networking, and Signal Processing Research Group and Interdisciplinary Center of Excellence in Telemedicine. His research interests are wireless communications, networking, and signal processing and its applications.

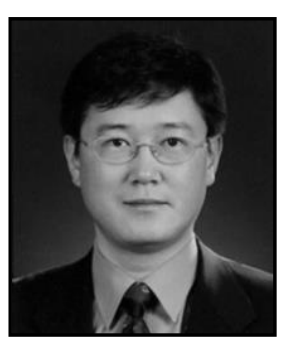

\section{In-Ho Ra}

$\mathrm{He}$ received the $\mathrm{Mc}$ and $\mathrm{PhD}$ egrees in computer engineering from Chung-Ang University, Seouf, Korea, in 1991 and 1995, respectively. He is currently a Professor at the Department of Information and Telecommunication Engineering, Kunsan National University, Kunsan, South Korea. From 2007 to 2008, he was a Visiting Scholar at the University of South Flonida, Tampa, Florida, USA. His research interests include mobile wireless communication networks, sensor networks, middleware design, cross-layer design, quality-of-service management of integrated sensor networks and social networks.

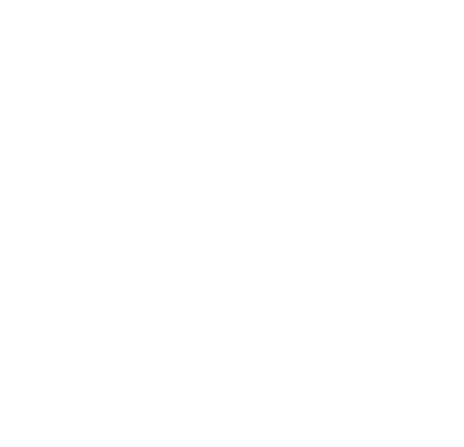


International Journal of Multimedia and Ubiquitous Engineering Vol.9, No.2 (2014)

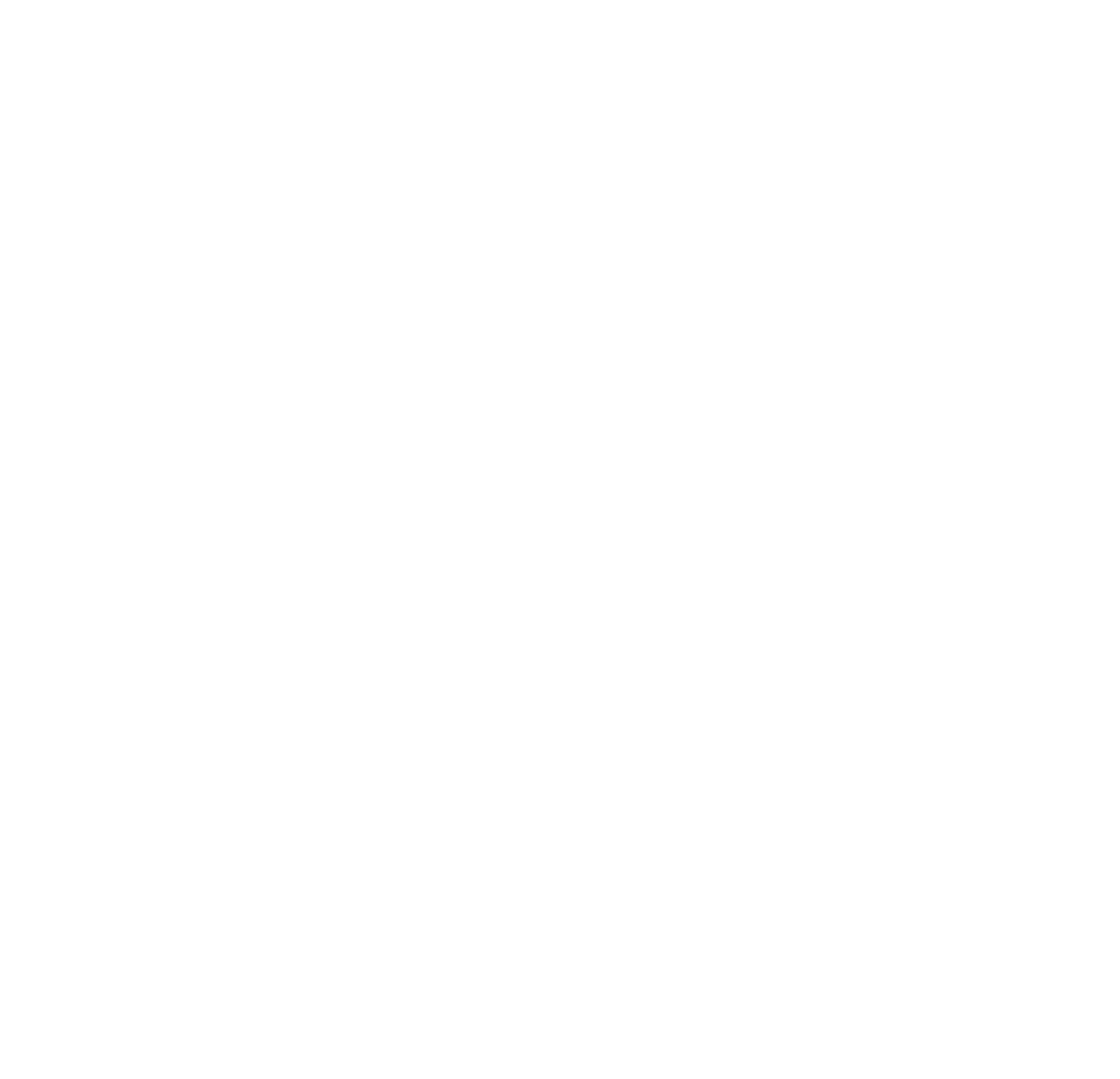

